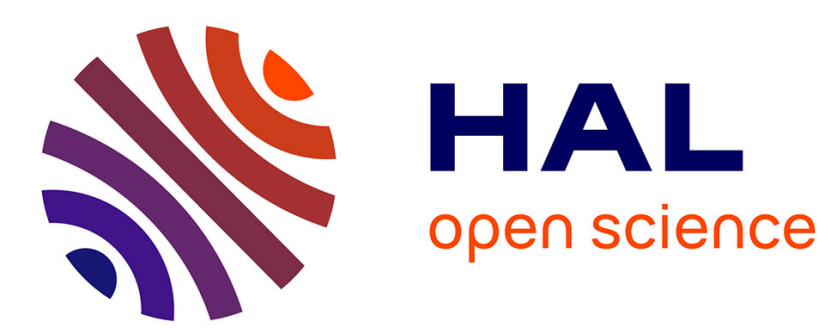

\title{
DISCUSSION DU MÉCANISME DES RÉACTIONS DE TRANSFERT TRĖS INÉLASTIQUE
}

\author{
R. Bimbot
}

\section{To cite this version:}

R. Bimbot. DISCUSSION DU MÉCANISME DES RÉACTIONS DE TRANSFERT TRÈS INÉLASTIQUE. Colloquium on Nuclear Surface, 1975, Dijon, France. pp.C5-53-C5-57, 10.1051/jphyscol:1975507 . jpa-00216362

\section{HAL Id: jpa-00216362 https://hal.science/jpa-00216362}

Submitted on 1 Jan 1975

HAL is a multi-disciplinary open access archive for the deposit and dissemination of scientific research documents, whether they are published or not. The documents may come from teaching and research institutions in France or abroad, or from public or private research centers.
L'archive ouverte pluridisciplinaire HAL, est destinée au dépôt et à la diffusion de documents scientifiques de niveau recherche, publiés ou non, émanant des établissements d'enseignement et de recherche français ou étrangers, des laboratoires publics ou privés. 


\title{
DISCUSSION DU MÉCANISME DES RÉACTIONS DE TRANSFERT TRÈS INÉLASTIQUE
}

\author{
R. BIMBOT \\ Chimie Nucléaire, Institut de Physique Nucléaire \\ BP. no 1,91406 Orsay, France
}

\begin{abstract}
Résumé. - Sous ce terme sont englobées les réactions qui mettent en jeu des échanges importants de matière et d'énergie, sans aboutir à la fúsion complète des partenaires. Lorsque le projectile utilisé est relativement léger $(A<40)$, on observe des réactions de transfert très inélastique dont les distributions angulaires dans le système du centre de masse correspondent à un produit léger émis préférentiellement vers l'avant, et pour des projectiles plus lourds, des réactions dites de quasi-fission dont les distributions angulaires présentent un maximum proche de l'angle d'effleurement. Les modèles théoriques utilisés pour rendre compte de ces réactions supposent l'intervention de forces de frottement (viscosité) entre les noyaux. Ces forces sont responsables des grandes pertes d'énergie cinétique observées. Le transfert de matière est traité comme un processus de diffusion en utilisant les équations de transfert issues de la mécanique statistique.
\end{abstract}

\begin{abstract}
This paper deals with reactions involving significant exchanges of matter or energy without leading to the complete fusion of the colliding nuclei. For relatively light projectiles $(A<40)$, one observes deep inelastic transfer reactions with angular distribution of the light residues peaking forward in the c.m. system, and for heavier projectiles, quasi-fission reactions for which the angular distributions exhibit a peak in the vicinity of the grazing angle. The theoretical models which are used to explain these reactions are based on the assumption of the existence of friction (or viscosity) forces which are responsible for the large losses of kinetic energy observed. The transfer of matter is treated as a diffusion process by using transport equations in the frame of statistical mechanics.
\end{abstract}

Pendant très longtemps, les études de réactions nucléaires induites par ions lourds ont été principalement consacrées à deux types de réactions : les réactions de transfert quasi élastique dans lesquelles l'interaction nucléaire est faible, et qui conduisent à des échanges de quelques nucléons, et des faibles pertes d'énergie cinétique, et les réactions de fusion complète, où les deux partenaires se réunissent en un seul noyau, sous l'effet d'une forte interaction nucléaire, avec transformation totale de l'énergie cinétique initiale en énergie d'excitation.

Les réactions que nous nous proposons de discuter correspondent à des pertes d'énergie importantes, tout en conduisant à des produits qui peuvent être proches du projectile et de la cible. Elles ont reçu des noms divers suivant les auteurs qui les ont étudiées. Cependant, on peut les classer en deux groupes qui se distinguent principalement par la distribution angulaire des produits de réaction :

- Les transferts très inélastiques [1-5], observés avec des projectiles légers $(A \leqslant 40)$ d'énergie élevée.

- Les réactions de quasi-fission [6-8] obtenues avec des projectiles lourds $(A>40)$ d'énergie peu supérieure à la barrière d'interaction.

Tout d'abord, nous illustrerons par quelques figures, montrant les résultats expérimentaux, les caracté- ristiques essentielles de ces deux types de réaction. Ensuite, nous discuterons qualitativement la façon dont on peut rendre compte de ces résultats par une approche macroscopique, dans le cadre de la théorie semi-classique, en introduisant des forces de frottement (ou de viscosité) nucléaire pour expliquer l'aspect dynamique de la réaction, et par un traitement de mécanique statistique (processus de diffusion) pour expliquer les transferts de matière.

1. Les faits expérimentaux. - 1.1 LES RÉACTIONS DE TRANSFERT TRÈS INÉLASTIQỤES. - 1.1.1 Les distributions angulaires dans le système C.M. - Elles correspondent à un produit léger émis préférentiellement vers l'avant, et à un produit lourd émis vers l'arrière. Cela apparaît nettement sur les figures 1 et 2 . La complémentarité de ces distributions angulaires est une bonne indication d'un processus à deux corps principaux dans la voie de sortie. Des mesures directes de corrélation angulaire des fragments [10] ont confirmé ce résultat.

1.1.2 Les distributions en énergie. - Elles mettent en évidence un ralentissement presque complet des deux partenaires au moment du choc. Ceci se remarque en particulier sur la figure 2 où l'on constate que le maximum des distributions en énergie cor- 


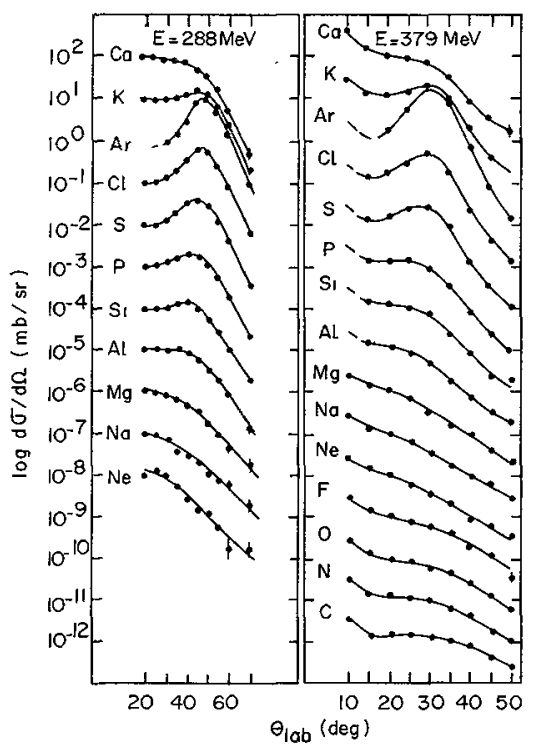

FIG. 1, - Distributions angulaires des produits légers correspondant aux transferts très inélastiques induits par $\mathrm{Ar}+\mathrm{Th}$ [4]. Afin de clarifier la figure, les courbes correspondant aux différents éléments ont été décalées verticalement de façon arbitraire.

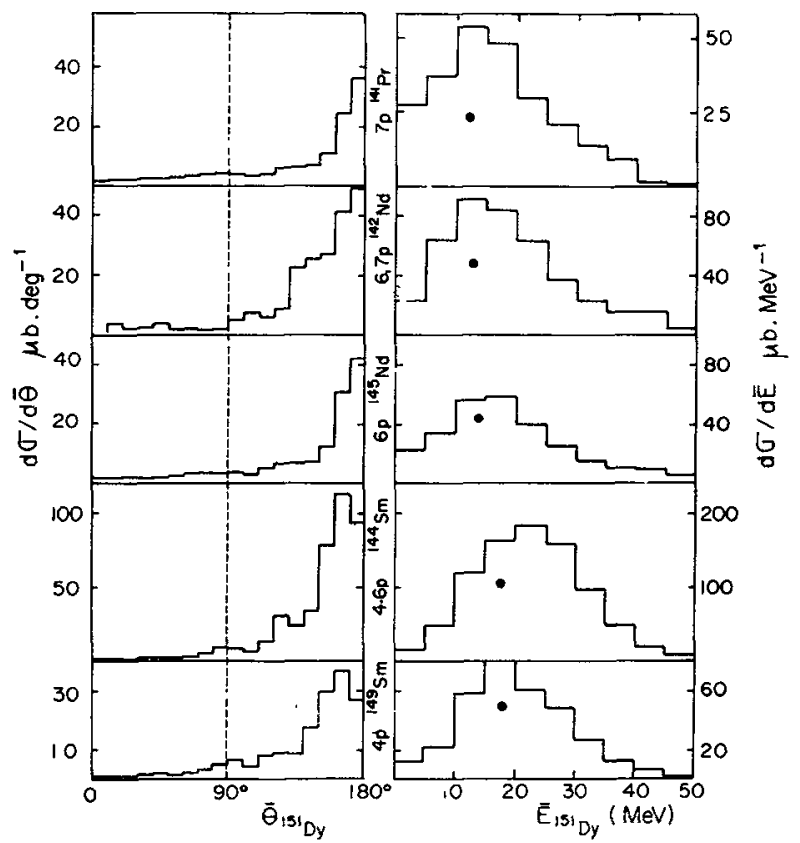

FIG. 2. - Distributions angulaires et énergétiques dans le système du centre de masse des produits lourds résultant de la capture de plusieurs charges par des cibles de terres rares, le projectile étant ${ }^{40} \mathrm{Ar}$ à $282 \mathrm{MeV}$ [9]. La nature de la cible et le nombre de charges transférées sont indiqués au centre. Pour chaque distribution énergétique, le point noir indique l'énergie correspondant à la répulsion coulombienne de deux sphères de rayon $1,6 \cdot \mathrm{A}^{1 / 3} \mathrm{Fm}$.

respond à la simple répulsion coulombienne des deux fragments au contact et au repos. L'énergie cinétique qui restait aux noyaux incidents au moment de l'interaction, après ralentissement par la répulsion électrostatique, a donc été entièrement convertie en énergie d'excitation des fragments.

Les mêmes résultats ont été trouvés par observation du résidu léger [11].
1.1.3 Le transfert de matière varie de quelques nucléons à plus de quinze nucléons quand le projectile est ${ }^{40} \mathrm{Ar}$. Il a été montré $[4,9]$ que les sections efficaces décroissent quand le nombre de charges transférées augmente. En outre, ce transfert correspond à l'équilibration du rapport $N / Z$ du nombre de neutrons au nombre de protons dans les deux produits de réaction [11].

1.2 LeS RÉACTIONS DE QUASI-FISSION. - L'utilisation de faisceaux d'ions très lourds $\left({ }^{84} \mathrm{Kr}\right)$ a montré que des transferts de grands nombres de nucléons apparaissent dès que la barrière d'interaction est franchie [6, 12].

Le phénomène de quasi-fission a été mis en évidence à Orsay $[6,7,13]$, par des mesures d'énergie effectuées en corrélation sur les deux produits de réaction, les ions incidents étant ${ }^{84} \mathrm{Kr}$ et ${ }^{63} \mathrm{Cu}$.

La figure 3 montre comment ce phénomène se distingue de la fission classique : cette figure représente la somme des énergies cinétiques des deux fragments en fonction de la masse de l'un d'eux (l'autre fragment ayant la masse complémentaire), et ceci pour deux types de réactions : la réaction ${ }^{40} \mathrm{Ar}+{ }^{238} \mathrm{U}$, le noyau de fusion étant l'isotope ${ }^{278} 110$ (figure du bas), et la réaction ${ }^{84} \mathrm{Kr}+{ }^{186} \mathrm{~W}$ pour laquelle le noyau de fusion serait ${ }^{270} 110$ (figure du haut). Les contours correspondent à des nombres d'événements égaux.

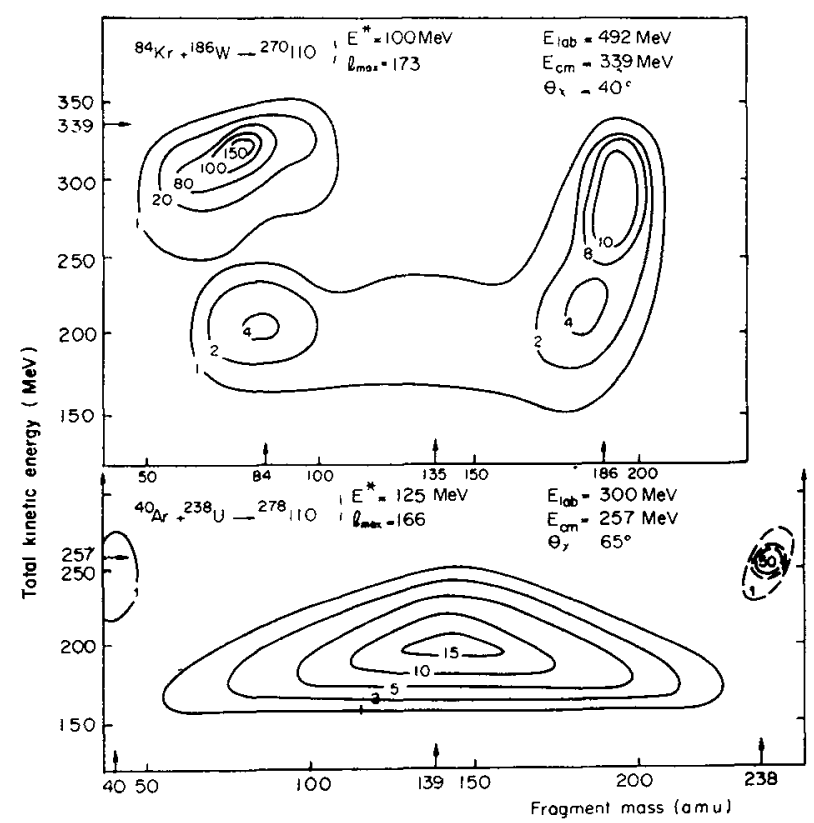

Fig. 3. - Fission et quasi-fission [13] (voir texte).

Sur la figure du bas, on constate la présence d'événements, d'une part pour les masses 40 et 238 avec une énergie cinétique proche de l'énergie initiale (événements quasi élastiques), d'autre part, dans une région de masse autour de la masse moyenne

$$
139=\frac{A_{\mathrm{Ar}}+A_{\mathrm{U}}}{2},
$$


avec une énergie de l'ordre de $200 \mathrm{MeV}$ (événements de fission symétrique suivant la fusion totale).

Sur la figure du haut, la situation est bien différente : on retrouve la présence d'événements quasi-élastiques, et la trace de rares événements de fission, mais il s'y ajoute en quantité significative des produits dont la masse est voisine de celles du projectile et de la cible, et dont l'énergie est proche de l'énergie de fission (énergie de répulsion coulombienne). Ce sont ces événements qui correspondent au processus de quasifission. Il est frappant de constater qu'ils n'apparaissent pas comme une traîne vers les basses énergies des événements de transfert quasi élastique, mais constituent un ensemble nettement distinct.

La figure 3 montre en outre que la section efficace de fusion totale (qui correspond à la fission symétrique) est importante pour les réactions $\mathrm{Ar}+\mathrm{U}$, et faible pour les réactions $\mathbf{K r}+\mathbf{W}$, qui conduisent pourtant à un noyau composé voisin. Il semble donc que la quasi-fission apparaisse en même temps que la probabilité de fusion complète diminue.

Sur la figure 4 est représentée la distribution angulaire des produits légers de quasi-fission [25]. On voit que cette distribution présente un maximum très prononcé pour un angle situé non loin de l'angle d'affleurement $\left(100^{\circ}\right.$ C.M.) ce qui la distingue nettement de celle des produits de transfert très inélastique (Fig. 1) et de celle des produits de fission qui est symétrique par rapport à $90^{\circ}$ C.M.

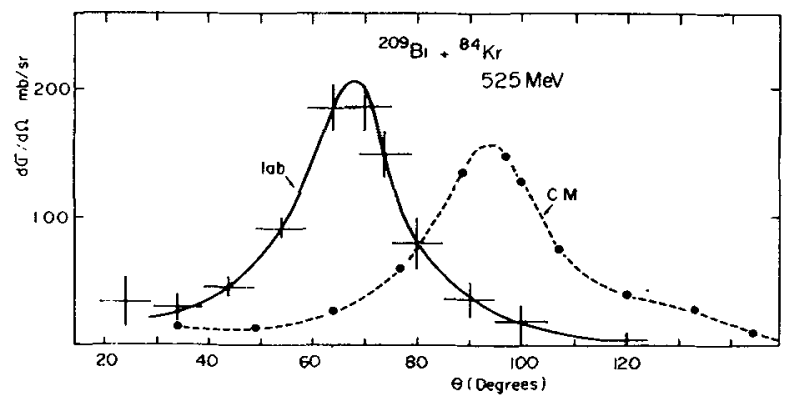

FIG. 4. - Distribution angulaire des produits de quasi-fission [25].

En résumé, les réactions de quasi-fission et de transfert très inélastiques ont des caractéristiques semblables du point de vue des masses transférées et des distributions en énergie, mais très différentes du point de vue des distributions angulaires.

Il faut cependant ajouter que des expériences très récentes semblent montrer qu'en utilisant soit des ions très lourds à plus haute énergie $(\mathrm{Kr}+\mathrm{Bi}$ à $715 \mathrm{MeV})$ [14] soit des ions de masse intermédiaire entre $\mathrm{Ar}$ et $\mathrm{Cu}(\mathrm{Fe}+\mathrm{Sm}$ à $375 \mathrm{MeV})$ [15], on obtient des distributions angulaires C.M., présentant à la fois un maximum proche de l'angle d'affleurement et une remontée vers l'avant en ce qui concerne les produits légers, et vers l'arrière pour les produits lourds.

2. Les approches théoriques. - Les résultats expérimentaux ci-dessus ont révélé que les noyaux peuvent perdre une très grande partie de leur énergie cinétique, c'est-à-dire subir une très forte interaction nucléaire, sans que cela les conduise obligatoirement à la fusion. On a donc été amené à penser que ces interactions se produisaient pour des valeurs de moment angulaire orbital $l$ assez élevées, et que la collision aboutirait à la formation d'un éphémère système composite qui, trop déformé pour être stable, se décomposerait en deux noyaux proches des produits initiaux, avant d'avoir eu le temps d'effectuer une révolution complète.

Les développements de ce modèle ont été faits principalement dans le cadre de la théorie semiclassique, c'est-à-dire que l'on considère que les noyaux ont des trajectoires bien définies. Le long de ces trajectoires agissent des forces conservatives (qui dérivent d'un potentiel) et non conservatives. Ces dernières sont responsables de la transformation de l'énergie cinétique en énergie d'excitation, et sont généralement traitées comme des forces de frottement (ou de viscosité) proportionnelles à la vitesse relative tangentielle ou radiale. Un certain nombre de travaux s'attachent à décrire ces phénomènes de friction d'un point de vue microscopique [16-18].

2.1 INTERPRÉTATION DES DISTRIBUTIONS ANGULAIRES. - Le potentiel traduisant l'interaction de deux noyaux à une distance $R$ est la somme d'un terme coulombien répulsif $V_{c}$, et d'un terme nucléaire attractif $V_{\mathrm{N}}$, auxquels on ajoute le terme centrifuge

$$
V_{\mathrm{ce}}=\frac{l(l+1) \hbar^{2}}{2 \mu R^{2}}
$$

où $\mu$ est la masse réduite, pour tenir compte du moment angulaire orbital :

$$
V=V_{\mathrm{c}}+V_{\mathrm{N}}+V_{\mathrm{ce}} .
$$

L'allure générale des potentiels d'interaction pour différentes valeurs de $l$ est représentée sur la figure $5 a$. Pour les faibles valeurs de $l$, et pour des couples projectiles-cibles ne correspondant pas à un produit $z_{1} z_{2}$ trop élevé, il existe un creux qui traduit l'influence attractive du potentiel nucléaire.

$\mathrm{Si}$ on simplifie le problème en considérant une simple diffusion inélastique (sans transfert de matière), et au cours de laquelle le moment angulaire serait conservé, on peut comprendre, sur la figure $5 a$, comment on obtiendra une perte plus ou moins grande d'énergie cinétique, associée à une perturbation plus ou moins grande de la trajectoire de Rutherford, suivant la valeur du moment angulaire initial :

- pour $l=l_{1}$ (faible), on aura une capture du projectile par la cible, c'est-à-dire fusion complète ;

- pour $l=l_{4}$, on aura une diffusion quasi élastique avec trajectoire quasi coulombienne (Fig. 5b) ;

- pour $l=l_{2}$ ou $l_{3}$, on aura une diffusion très inélastique avec une trajectoire fortement perturbée 


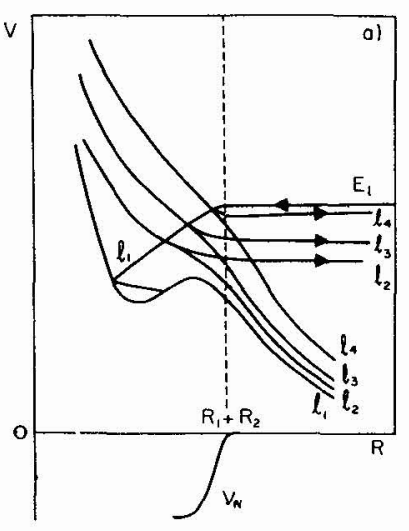

b)

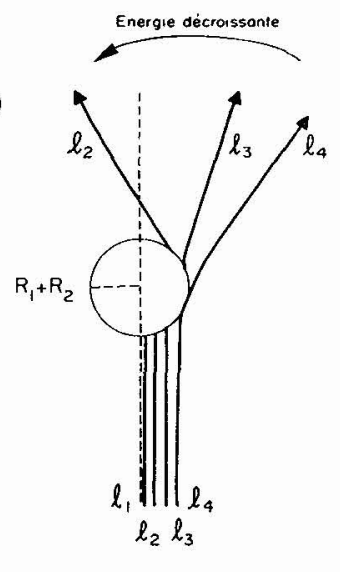

FIG. 5a. - Potentiel d'interaction et pertes d'énergie en fonction du moment angulaire $l$.

FIG. 5b. - Trajectoires et pertes d'énergie d'après [19] (voir texte).

par l'attraction nucléaire. Cette trajectoire pourra aboutir à un angle d'émission positif $\left(l_{3}\right)$ ou négatif $\left(l_{2}\right)$ de la particule diffusée (Fig. $5 b$ ).

Suivant ce schéma proposé par Wilczinsky [19], la perte d'énergie serait d'autant plus grande que l'angle de déflection $\theta(l)-\theta\left(l_{4}\right)$ serait plus grand. La contribution des angles positifs et négatifs s'ajoutant, on expliquerait ainsi l'observation, pour certains angles, de deux pics dans la distribution en énergie. $\mathrm{Au}$ voisinage de $0^{\circ}$, ces deux pics fusionneraient en un seul, et on pourrait alors observer un maximum proche de $0^{\circ}$ dans la distribution angulaire.

Cette représentation est en bon accord avec les résultats obtenus à Dubna et présentés sur la figure 6, pour les transferts très inélastiques [19]. Il est bien évident qu'il ne s'agit plus ici de diffusion inélastique, et que les transferts de matière et de moment angulaire devraient être pris en compte, mais la figure 6 montre que le schéma donné explique qualitativement les résultats obtenus. Des calculs effectués par Gross et al. [20] confirment ce résultat.

Lorsque les numéros atomiques des noyaux en collision augmentent, la répulsion coulombienne a tendance à faire disparaître le creux de potentiel, et de grandes pertes d'énergie peuvent se produire dans une région où le potentiel d'interaction reste fortement répulsif. On pourrait expliquer ainsi [20, 24] les maxima observés non loin de l'angle d'affleurement pour les réactions de quasi-fission.

2.2 Le TRANSFeRT De MATIÈRE. - Pour rendre compte du transfert de nucléons, on considère géné-

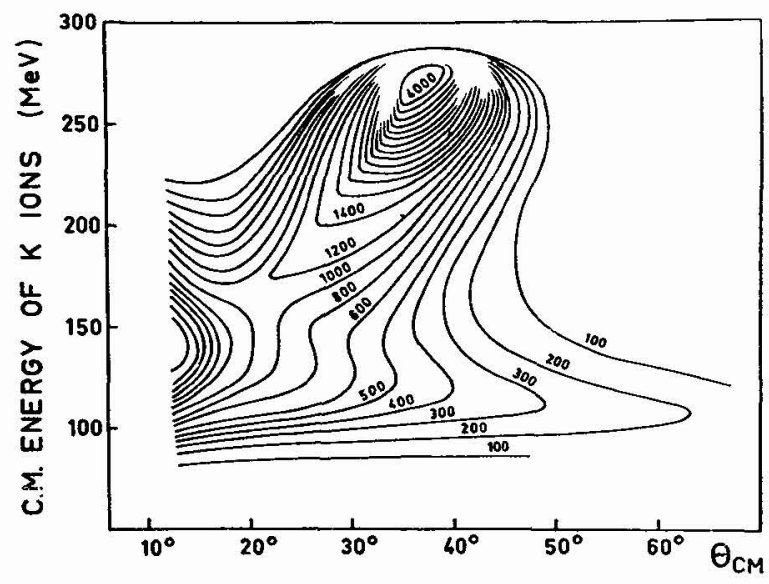

Fig. 6. - Lignes d'égale section efficace différentielle (en $\mu \mathrm{b} / \mathrm{MeV}$.rad) de la réaction ${ }^{232} \mathrm{Th}\left({ }^{40} \mathrm{Ar}, \mathrm{K}\right)$ à $388 \mathrm{MeV}$, en fonction de l'angle d'observation ${ }_{\text {C.M. }}$ et de l'énergie C.M. des ions K [19].

ralement l'ensemble (noyau composite) formé par les deux noyaux en contact au moment de l'interaction, et on admet que les nucléons vont difiuser d'un noyau vers l'autre à la façon dont les molécules de gaz ou de liquides peuvent diffuser lorsqu'on met deux fluides en contact.

Nörenberg [21] a montré que les équations de transport de la mécanique statistique peuvent être utilisées pour calculer l'évolution des distributions en énergie ou des distributions en $Z$ des produits de transfert très inélastique lorsque l'angle d'observation varie. Dans ce calcul, le temps d'interaction est déduit de l'angle de déflection, et les coefficients caractérisant la diffusion sont déterminés par la comparaison avec l'expérience.

Une approche légèrement différente a été utilisée par Moretto et Sventek [22] qui d'une part simplifient la partie dynamique du calcul, et d'autre part font intervenir la variation de l'énergie potentielle en fonction de l'asymétrie de charge dans le noyau composite pour le calcul statistique de la diffusion. Ils parviennent ainsi à reproduire de façon satisfaisante les distributions angulaires des éléments produits dans les réactions ${ }^{40} \mathrm{Ar}+\mathrm{Ag}$ à $288 \mathrm{MeV}$.

Dans ces calculs, les durées d'interaction sont de l'ordre de $10^{-21} \mathrm{~s}$, c'est-à-dire environ 10 fois plus longues que le temps mis par un nucléon de $40 \mathrm{MeV}$ pour traverser le noyau. Un grand nombre de nucléons peuvent donc être impliqués dans l'interaction, ce qui justifie l'emploi de méthodes statistiques, et qui rapproche ce type de réactions des réactions de fusion. C'est pourquoi le terme de fusion incomplète a été proposé [23] pour désigner l'ensemble de ces réactions. 


\section{Bibliographie}

[1] Kaufmann, R. et Wolfgang, R., Phys. Rev. 121 (1960) 192.

[2] Galin, J., Guerreau, D., Lefort, M., Peter, J., Tarrago, X., et Basile, R., Nucl. Phys. A 159 (1970) 461.

[3] Artukh, A. G., Gridnev, G. F., Mikheev, V. L., Volkov, V. V., Wilczinsky; J., Nucl: Phys. A 211 (1973) 299.

[4] Artukh, A. G., Gridnev, G. F., Mrkheev, V. L., Volkov, V. V., Wilczinsky, J., Nucl. Phys. A 215 (1973) 91.

[5] Jacmart, J. C., Colombani, P., Doubre, H., Frascaria, N., Poffé, N., Riou, M., Roynette, J. C., Stephan, C., WeIDINGer, A., Nucl. Phys. A 242 (1975) 175;

voir aussi Jacmart, J. C., Frascaria, N., Poffé, N., Colombani, P., Doubre, H., Riou, M., Roynette, J. C., STÉPHan, C., J. Physique Colloq. 36 (1975) C.

[6] Lefort, M., Ngo, C., Peter, J., Tamain, B., Nucl. Phys. A 216 (1973) 166.

[7] Hanappe, F., Lefort, M., NGo, G., Peter, J., et Tamain, B., Phys. Rev. Lett. 32 (1974) 738.

[8] Wolf, K. L., UNiK, J. P., Huizenga, J. R., Birkelund, J., Freisleben, H., Viola, V. E., Phys. Rev. Lett. 33 (1974) 1105.

[9] Bimbot, R., Gardes, D., HaHn, R. L., De Moras, Y., Rivet, M. F., Rapport IPNO-RC-75 01, Nucl. Phys. A 248 (1975) 377.

[10] Thompson, S. G., Moretto, L. G., Jared, R. C., Babinet, R. P., Galin, J., Fowler, M. M., Gatti, R. C., Hunter, J. B., Nobel Symposium on super heavy elements, Ronneby, Phys. Script. (1974) sous presse.

[11] Gatty, B., Guerreau, D., Lefort, M., Pouthas, J., Tarrago, X., Galin, J., Cauvin, B., Girard, J., Nifenecker,
H., J. Physique-Lett. 35 (1974) L-117 et Z. Phys. A 275 (1975) 65.

[12] Bimbot, R., Gauvin, H., Le Beyec, Y., Lefort, M., Porile, N. T., Tamain, B., Nucl. Phys. A 189 (1972) 539.

[13]·Peter, J., HanapPe, F., NGo, C., Tamain, B., Rapport IPNORC-73 07 et Proc. Int. Conf. Nucl. Phys. Munich vol 1 (1973) 611.

[14] HutZenga, J. R. et al. (résultats non publiés).

[15] Rivet, M. F., Thèse en préparation (Orsay) non publié.

[16] Glas, D., Mosel, U., Phys. Lett. 49B (1974) 301.

[17] Gross, D. H. E., Nucl. Phys. A 240 (1975) 412.

[18] Giraud, B., Le Tourneux, J., Osnes, E., Phys. Rev. C 11 (1975) 82.

[19] WiLCZINSKY, J., Phys. Lett. B 47 (1973) 484

[20] Gross, D. H. E., Kalrnowski, H., DE, J. N., Symposium on classical, and quantum mechanical aspects of H. I. collisions, Heidelberg (1974)

[21] Norenderg, W., Phys. Lett. 53B (1974) 289.

[22] MoRetTo, L. G., SventeK, J. S., Report, LBL. 3443 (1974) (soumis à Phys. Lett.).

[23] LeFORT, M., Europhysics Conference on Nuclear Interactions at Medium and Low Energies, Harwell March 24-26 (1975).

[24] Bondorf, J. P., Huizenga, J. R., Sobel, M. I., Sperber, D., Phys. Rev. C 11 (1975) 1265.

[25] Hanappe, F., Ngo, C., Peter, J., Tamain, B., Proc. Int. Conf. Reactions between Complex Nuclei, Nashville, vol. 1 (1974) 116. Ed. by Robinson et al. (North-Holland). 